\title{
Oscillation Criteria of Second-Order Mixed Neutral Delay Dynamic Equations on Time Scales
}

\author{
L M Feng, Y G Zhao, Y L Shi and Z L Han ${ }^{a}$ \\ School of Mathematical, University of Jinan, Jinan, Shandong 250022, P R China
}

Abstract. In this artical, we consider a second-order neutral dynamic equation on a time scales. A number of oscillation theorems are shown that supplement and extend some known results in the eassay.

\section{Introduction}

In this essay, we will consider the oscillation of the second-order mixed neutral delay dynamic equation

$$
\left(r(n) x^{\Delta}(n)\right)^{\Delta}+q(n) y(\delta(n))=0, n \in\left[n_{0}, \infty\right)_{T},
$$

where $x(n):=y(n)+a(n) y\left(n-\tau_{1}\right)+b(n) y\left(n+\tau_{2}\right), \tau_{1}$ and $\tau_{2}$ are nonnegative constants, $n_{0} \in T$ and $\left[n_{0}, \infty\right)_{T}$ $:=\left[n_{0}, \infty\right) \cap T$ denotes a time scale interval with sup $T=\infty$. We shall assume the following conditions: (C1) $a, b, q \in C_{r d}\left(\left[n_{0}, \infty\right)_{T}, R\right)$, where

$$
\begin{gathered}
0 \leq a(n) \leq a_{0}<\infty, 0 \leq b(n) \leq b_{0}<\infty, \text { and } q(n)>0 . \\
(\mathrm{C} 2) r \in C_{r d}\left(\left[n_{0}, \infty\right)_{T}, R\right), r(n)>0, \int_{n_{0}}^{\infty} \frac{1}{r(n)} \Delta n<\infty . \\
\text { (C3) } \delta \in C_{r d}\left(\left[n_{0}, \infty\right)_{T}, T\right), \delta^{\Delta}(n)>0, \delta(n) \leq n, \\
\lim _{t \rightarrow \infty} \delta(n)=\infty .
\end{gathered}
$$

Neutral functional difference and differential equations have many applications in many fields, like electric networks. As an important part, difference and differential equations with mixed type neutral term have attracted many researcher's attention, see [1-4].

Han et al. [1] investigated the equations of mixed type

$$
\begin{aligned}
& {\left[\left(y(n)+p_{1} y\left(n-\tau_{1}\right)+p_{2} y\left(n+\tau_{2}\right)\right)^{\gamma}\right]^{\prime \prime}} \\
& =q_{1}(n) y^{\gamma}\left(n-\sigma_{1}\right)+q_{2}(n) y^{\gamma}\left(n+\sigma_{2}\right),
\end{aligned}
$$

where $\gamma \geq 1$ is the ratio of positive odd numbers. Some oscillation theorems are established and the previous results are generalized.

The theory of time scale, which has received numerous attention, was introduced by Hilger's landmark contribution article [5], in order to unify discrete and continuous calculus theory, and it also extends these classical cases to cases 'in between'. A time scale $T$ is an arbitrary nonempty closed subset of the real numbers field $R$.

The oscillation of solutions of various dynamic equations on time scales has attracted wide attention in the last several years. For the oscillation research on time scales, readers can refer to the [6-11], and the references cited therein. There are a few results regarding the oscillation of second order neutral dynamic equations with mixed neutral term

Our objective of this paper is establish some new criteria for the more generalized second order neutral dynamic equation (1), which also allow to relax some restrictive conditions imposed on the equation.

If there are infinitely large generalized zeros on $\left[T_{y}, \infty\right)_{T}$, then the solution $y$ of equation (1) is oscillatory; otherwise, it is called nonoscillatory. Equation (1) is said to be oscillatory if all its solutions are oscillatory.

\section{Main results}

Theorem 1. Assume that $\delta(n \pm \alpha)=\delta(n) \pm \alpha(\alpha>0)$. If

$$
\int_{n_{0}}^{\infty} Q(s) \Delta s=\infty,
$$

and

$$
\limsup _{n \rightarrow \infty} \int_{n_{0}}^{n}\left[\phi(s) Q(s)-\frac{1+a_{0}+b_{0}}{4 r(s) \phi(s)}\right] \Delta s=\infty,
$$

where

$$
Q(n)=\min \left\{q(n), q\left(n-\tau_{1}\right), q\left(n+\tau_{2}\right)\right\}, \phi(n)=\int_{n}^{\infty} \frac{1}{r(s)} \Delta s .
$$

Then equation (1) oscillation.

Proof. Suppose that $x$ is a eventually positive

\footnotetext{
a Corresponding author: hanzhenlai@163.com
} 
solution of (1). Yet the general, we may assume that $n_{1} \geq n_{0}$ exists to make $y(n), y\left(n-\tau_{1}\right), y\left(n+\tau_{2}\right)$ and $y(\delta(n))$ are all positive for $n \in\left[n_{1}, \infty\right)_{T}$.

Then we have $x(n)>0$. We get that $r(n) x^{\Delta}(n)<0$ from (1), then $x^{\Delta}(n)$ one sign at an end. Hence, there exist two cases for $n \in\left[n_{1}, \infty\right)_{T}$, that is,

(i) $x(n)>0, x^{\Delta}(n)>0,\left(r(n) x^{\Delta}(n)\right)^{\Delta} \leq 0$;

(ii) $x(n)>0, x^{\Delta}(n)<0,\left(r(n) x^{\Delta}(n)\right)^{\Delta} \leq 0$.

Firstly, assume that case (i) hold. Applying (1), we have

$$
\begin{gathered}
\left(r(n) x^{\Delta}(n)(n)\right)^{\Delta}+q(n) y(\delta(n))+a_{0}\left(r\left(n-\tau_{1}\right) x^{\Delta}\left(n-\tau_{1}\right)\right)^{\Delta} \\
+a_{0} q\left(n-\tau_{1}\right) y\left(\delta\left(n-\tau_{1}\right)\right)+b_{0}\left(r\left(n+\tau_{2}\right) x^{\Delta}\left(n+\tau_{2}\right)\right)^{\Delta} \\
+b_{0} q\left(n+\tau_{2}\right) y\left(\delta\left(n+\tau_{2}\right)\right)=0 .
\end{gathered}
$$

From $y(n)$ and $Q(n)$, we obtain

$$
\begin{gathered}
\left(r(n) x^{\Delta}(n)\right)^{\Delta}+a_{0}\left(r\left(n-\tau_{1}\right) x^{\Delta}\left(n-\tau_{1}\right)\right)^{\Delta} \\
+b_{0}\left(r\left(n+\tau_{2}\right) x^{\Delta}\left(n+\tau_{2}\right)\right)^{\Delta}+Q(n) y(\delta(n)) \leq 0 .
\end{gathered}
$$

Integrating (4) from $n_{2}$ to $n$, and from $x(n) \geq c>0$ ( $c$ is a constant) for $n \in\left[n_{2}, \infty\right)_{T}$, we obtain

$$
\begin{aligned}
c \int_{n_{2}}^{n} Q(s) \Delta s & \leq r\left(n_{2}\right) x^{\Delta}\left(n_{2}\right)+a_{0} r\left(n_{2}-\tau_{1}\right) x^{\Delta}\left(n_{2}-\tau_{1}\right) \\
& +b_{0} r\left(n_{2}+\tau_{2}\right) x^{\Delta}\left(n_{2}+\tau_{2}\right),
\end{aligned}
$$

which conflict with (2).

Next, suppose that case (ii) hold. Define a function $\omega$ by

$$
\omega(n)=\frac{r(n) x^{\Delta}(n)}{x(n)}, n \in\left[n_{2}, \infty\right)_{T} .
$$

Obviously, $\quad \omega(n)<0$. Since $r(n) x^{\Delta}(n) \quad$ is decreasing, we have

$$
x(l) \leq x(n)+r(n) x^{\Delta}(n) \int_{n}^{l} \frac{1}{r(s)} \Delta s, l \geq n \geq n_{2} .
$$

When $l \rightarrow \infty$, we conclude

$$
x(n)+r(n) x^{\Delta}(n) \phi(n) \geq 0, n \in\left[n_{2}, \infty\right)_{T} .
$$

Therefore, we can obtain

$$
\frac{r(n) x^{\Delta}(n)}{x(n)} \phi(n) \geq-1, n \in\left[n_{2}, \infty\right)_{T},
$$

and

$$
-1 \leq \omega(n) \phi(n) \leq 0, n \in\left[n_{2}, \infty\right)_{T} .
$$

Define $u$ as

$$
u(n)=\frac{r\left(n-\tau_{1}\right) x^{\Delta}\left(n-\tau_{1}\right)}{x(n)}, n \in\left[n_{2}, \infty\right)_{T} .
$$

Similarly, we know that $u(n)<0$, and from $r(n) x^{\Delta}(n)<0$, we have $r\left(n-\tau_{1}\right) x^{\Delta}\left(n-\tau_{1}\right) \geq r(n) x^{\Delta}(n)$. Then, $u(n) \geq \omega(n)$, because of (6), we get

$$
-1 \leq u(n) \phi(n) \leq 0, n \in\left[n_{2}, \infty\right)_{T} .
$$

Next, define function $v$ by

$$
v(n)=\frac{r\left(n+\tau_{2}\right) x^{\Delta}\left(n+\tau_{2}\right)}{x(n)}, n \in\left[n_{2}, \infty\right)_{T} .
$$

We know that $v(n)<0$, and since $r(n) x^{\Delta}(n)$ is decreasing, we have

$$
r(s) x^{\Delta}(s) \leq r\left(n+\tau_{2}\right) x^{\Delta}\left(n+\tau_{2}\right), s \geq n+\tau_{2} \geq n_{2} .
$$

Through calculation, we have

$$
\begin{gathered}
x(l) \leq x(n)+r\left(n+\tau_{2}\right) x^{\Delta}\left(n+\tau_{2}\right) \int_{n}^{l} \frac{1}{r(s)} \Delta s, \\
l \geq n+\tau_{2} \geq n_{2} .
\end{gathered}
$$

Letting $l \rightarrow \infty$, and from (9), then

$$
-1 \leq v(n) \phi(n) \leq 0, n+\tau_{2} \geq n_{2} .
$$

Differentiating (5), we obtain

$$
\begin{gathered}
\omega^{\Delta}(n)=\frac{\left(r(n) x^{\Delta}(n)\right)^{\Delta}}{x(\sigma(n))}-\frac{r(n)\left(x^{\Delta}(n)\right)^{2}}{z(n) x(\sigma(n))} \\
\leq \frac{\left(r(n) x^{\Delta}(n)\right)^{\Delta}}{x(\sigma(n))}-\frac{r(n)\left(x^{\Delta}(n)\right)^{2}}{x^{2}(n)} \\
=\frac{\left(r(n) x^{\Delta}(n)\right)^{\Delta}}{x(\sigma(n))}-\frac{\omega^{2}(n)}{r(n)}<0, n \geq n_{3} \geq n+\tau_{2} .
\end{gathered}
$$

Differentiating (7) and (9), as the similar process of (11), we have

$$
u^{\Delta}(n) \leq \frac{\left(r\left(n-\tau_{1}\right) x^{\Delta}\left(n-\tau_{1}\right)\right)^{\Delta}}{x(\sigma(n))}-\frac{u^{2}(n)}{r(n)}<0, n \in\left[n_{3}, \infty\right)_{T} .
$$

And

$$
v^{\Delta}(n) \leq \frac{\left(r\left(n+\tau_{2}\right) x^{\Delta}\left(n+\tau_{2}\right)\right)^{\Delta}}{x(\sigma(n))}-\frac{v^{2}(n)}{r(n)}<0, n \in\left[n_{3}, \infty\right)_{T} .
$$

Combining with (11), (12) and (13), we get

$$
\omega^{\Delta}(n)+a_{0} u^{\Delta}(n)+b_{0} v^{\Delta}(n)
$$

$\leq \frac{\left(r(n) x^{\Delta}(n)\right)^{\Delta}}{x(\sigma(n))}+\frac{a_{0}\left(r\left(n-\tau_{1}\right) x^{\Delta}\left(n-\tau_{1}\right)\right)^{\Delta}}{x(\sigma(n))}+\frac{b_{0}\left(r\left(n+\tau_{2}\right) x^{\Delta}\left(n+\tau_{2}\right)\right)^{\Delta}}{x(\sigma(n))}$ 


$$
-\frac{\omega^{2}(n)}{r(n)}-a_{0} \frac{u^{2}(n)}{r(n)}-b_{0} \frac{v^{2}(n)}{r(n)}, n \in\left[n_{3}, \infty\right)_{T} .
$$

Since, $x(n)$ is decreasing, from (4) and (13), we can obtain

$$
\begin{gathered}
\omega^{\Delta}(n)+a_{0} u^{\Delta}(n)+b_{0} v^{\Delta}(n) \\
\leq-Q(n)-\frac{\omega^{2}(n)}{r(n)}-a_{0} \frac{u^{2}(n)}{r(n)}-b_{0} \frac{v^{2}(n)}{r(n)}, n \in\left[n_{3}, \infty\right)_{T} .
\end{gathered}
$$

Multiplying both side of (15) by $\phi(n)$ and integrating from $n_{3}$ to $n$, using the integration by parts on time scales, we have

$$
\begin{gathered}
\phi(n) \omega(n)-\phi\left(n_{3}\right) \omega\left(n_{3}\right)-\int_{n_{3}}^{n} \phi^{\Delta}(s) \omega(\sigma(s)) \Delta s+\int_{n_{3}}^{n} \frac{\phi(s) \omega^{2}(s)}{r(s)} \Delta s \\
+a_{0} \phi(n) u(n)-a_{0} \phi\left(n_{3}\right) u\left(n_{3}\right)-a_{0} \int_{n_{3}}^{n} \phi^{\Delta}(s) u(\sigma(s)) \Delta s+a_{0} \int_{n_{3}}^{n} \frac{\phi(s) u^{2}(s)}{r(s)} \Delta s \\
\left.+b_{0} \phi(n) v(n)-b_{0} \phi\left(n_{3}\right)\right)\left(n_{3}\right)-b_{0} \int_{n_{3}}^{n} \phi^{\Delta}(s) v(\sigma(s)) \Delta s+b_{0} \int_{n_{3}}^{n} \frac{\phi(s) v^{2}(s)}{r(s)} \Delta s \\
+\int_{n_{3}}^{n} \phi(s) Q(s) \Delta s \leq 0 .
\end{gathered}
$$

Noting $\omega(n), u(n)$ and $v(n)$ are decreasing, from (16), we obtain

$$
\begin{gathered}
\phi(n) \omega(n)-\phi\left(n_{3}\right) \omega\left(n_{3}\right)-\int_{n_{3}}^{n} \frac{\omega(s)}{r(s)} \Delta s+\int_{n_{3}}^{n} \frac{\phi(s)}{r(s)} \omega^{2}(s) \Delta s \\
+a_{0} \phi(n) u(n)-a_{0} \phi\left(n_{3}\right) u\left(n_{3}\right)-a_{0} \int_{n_{3}}^{n} \frac{u(s)}{r(s)} \Delta s+a_{0} \int_{n_{3}}^{n} \frac{\phi(s)}{r(s)} u^{2}(s) \Delta s \\
+b_{0} \phi(n) v(n)-b \phi\left(n_{3}\right) v\left(n_{3}\right)-b_{0} \int_{n_{3}}^{n} \frac{v(s)}{r(s)} \Delta s+b_{0} \int_{n_{3}}^{n} \frac{\phi(s)}{r(s)} v^{2}(s) \Delta s \\
+\int_{n_{3}}^{n} \phi(s) Q(s) \Delta s \leq 0 .
\end{gathered}
$$

Using the averaging technique for (17), we obtain

$$
\begin{gathered}
\phi(n) \omega(n)-\phi\left(n_{3}\right) \omega\left(n_{3}\right)+a_{0} \phi(n) u(n)-a_{0} \phi\left(n_{3}\right) u\left(n_{3}\right) \\
+b_{0} \phi(n) v(n)-b_{0} \phi\left(n_{3}\right) v\left(n_{3}\right) \\
-\frac{1+a_{0}+b_{0}}{4} \int_{n_{3}}^{n} \frac{1}{r(s) \phi(s)} \Delta s+\int_{n_{3}}^{n} \phi(s) Q(s) \Delta s \leq 0 .
\end{gathered}
$$

Combining (6), (8) and (10) with (18), we have

$$
\begin{gathered}
\int_{n_{3}}^{n}\left[\phi(s) Q(s)-\frac{1+a_{0}+b_{0}}{4 r(s) \phi(s)}\right] \Delta s \\
\leq\left(1+a_{0}+b_{0}\right)+\phi\left(n_{3}\right)\left(\omega\left(n_{3}\right)+a_{0} u\left(n_{3}\right)+b_{0} v\left(n_{3}\right)\right),
\end{gathered}
$$

which conflict with (3). This proof is completes.

In next theorem, we need the following definition:

We say that a function $\varphi=\varphi(n, s, l) \in Y$, if $\varphi \in C(E, R)$, where $E=\left\{(n, s, l): n_{0} \leq l \leq s \leq n<\infty\right\}$, which satisfies $\varphi(n, n, l)=0, \varphi(n, l, l)=0 \quad$ and $\varphi(n, s, l)>0$ for $l<s<n$, and has a nonnegative rd- continuous $\Delta$-partial derivative $\varphi^{\Delta_{s}}$ on $E$ that it is locally integrable with respect to $S$ in $E$. Define the operator $T$ by

$$
T[g ; l, n]=\int_{l}^{n} \varphi(n, s, l) g(s) \Delta s,
$$

for $\quad n \geq s \geq l \geq t_{0} \quad$ and $\quad g \in C_{r d}^{1}\left[n_{0}, \infty\right)_{T}$. $\psi=\psi(n, s, l)$ is defined by

$$
\varphi^{\Delta_{s}}(n, s, l)=\psi(n, s, l) \varphi(n, s, l) .
$$

Then, $T$ is a linear operator obviously, and

$$
T\left[g^{\Delta} ; l, n\right]=-T\left[\psi g^{\sigma} ; l, n\right] .
$$

Theorem 2. Assume that $\delta(n \pm \alpha)=\delta(n) \pm \alpha(\alpha>0)$ and $\delta(n) \leq n-\tau_{1}$. If there is positive function $\eta \in C_{r d}^{1}\left(\left[n_{0}, \infty\right)_{T}, R\right)$ such that for $\varphi \in Y$ we have

$\limsup _{n \rightarrow \infty} T\left[\eta(s) Q(s)-\frac{\left(1+a_{0}+b_{0}\right)}{4 \eta(s)}(\psi(n, s, l)\right.$

$$
\left.\left.+\frac{\eta^{\Delta}(s)}{\eta(\sigma(s))}\right)^{2} r(\delta(s)) \eta^{2}(\sigma(s)) ; l, n\right]>0
$$

and

$$
\limsup T\left[Q(s)-\frac{\left(1+a_{0}+b_{0}\right)}{4} \psi^{2}(n, s, l) r(s) ; l, n\right]>0,
$$

where $Q(n)$ is shown in Th $1, T$ defined as (19), and $\psi=\psi(n, s, l)$ is shown by (20). Then equation (1) oscillation.

Proof. Suppose that $x$ is a nonoscillatory solution of (1). Yet the general, we may assume that $n_{1} \geq n_{0}$ exists to make $y(n), y\left(n-\tau_{1}\right), y\left(n+\tau_{2}\right)$ and $y(\delta(n))$ are positive for $n \in\left[n_{1}, \infty\right)_{T}$.

Proof steps similar to Theorem 1, for $n \in\left[n_{1}, \infty\right)_{T}$ we have case (i) and case (ii).

First assume that case (i) hold. Define

$$
\omega(n)=\eta(n) \frac{r(n) x^{\Delta}(n)}{x(\delta(n))}, n \in\left[n_{1}, \infty\right)_{T} .
$$

Obviously $\omega(n)>0$, and we have

$\omega^{\Delta}(n)$

$$
\begin{aligned}
& =\eta(n) \frac{\left(r(n) x^{\Delta}(n)\right)^{\Delta}}{x(\delta(n))}+\frac{\eta^{\Delta}(n)}{\eta(\sigma(n))} \omega(\sigma(n))-\frac{\eta(n) x^{\Delta}(\delta(n))}{\eta(\sigma(n)) x(\delta(n))} \omega(\sigma(n)) \\
& \leq \eta(n) \frac{\left(r(n) x^{\Delta}(n)\right)^{\Delta}}{x(\delta(n))}+\frac{\eta^{\Delta}(n)}{\eta(\sigma(n))} \omega(\sigma(n))-\frac{\eta(n)}{r(\delta(n)) \eta^{2}(\sigma(n))} \omega^{2}(\sigma(n)) .
\end{aligned}
$$

Define function 


$$
u(n)=\eta(n) \frac{r\left(n-\tau_{1}\right) x^{\Delta}\left(n-\tau_{1}\right)}{x(\delta(n))}, n \in\left[n_{1}, \infty\right)_{T} .
$$

Obviously $u(n)>0$, and we have

$$
\begin{aligned}
& u^{\Delta}(n) \\
& =\eta(n) \frac{\left(r\left(n-\tau_{1}\right) x^{\Delta}\left(n-\tau_{1}\right)\right)^{\Delta}}{x(\delta(n))}+\frac{\eta^{\Delta}(n)}{\eta(\sigma(n))} u(\sigma(n))-\frac{\eta(n) x^{\Delta}(\delta(n))}{\eta(\sigma(n)) x(\delta(n))} u(\sigma(n)) \\
& \leq \eta(n) \frac{\left(r\left(n-\tau_{1}\right) x^{\Delta}\left(n-\tau_{1}\right)\right)^{\Delta}}{x(\delta(n))}+\frac{\eta^{\Delta}(n)}{\eta(\sigma(n))} u(\sigma(n))-\frac{\eta(n)}{r(\delta(n)) \eta^{2}(\sigma(n))} u^{2}(\sigma(n)) .
\end{aligned}
$$

Define function

$$
v(n)=\eta(n) \frac{r\left(n+\tau_{2}\right) x^{\Delta}\left(n+\tau_{2}\right)}{x(\delta(n))}, n \in\left[n_{1}, \infty\right)_{T} .
$$

Obviously $u(n)>0$, and we have

$v^{\Delta}(n)$

$=\eta(n) \frac{\left(r\left(n+\tau_{2}\right) x^{\Delta}\left(n+\tau_{2}\right)\right)^{\Delta}}{x(\delta(n))}+\frac{\eta^{\Delta}(n)}{\eta(\sigma(n))} v(\sigma(n))-\frac{\eta(n) x^{\Delta}(\delta(n))}{\eta(\sigma(n)) x(\delta(n))} v(\sigma(n))$

$\leq \eta(n) \frac{\left(r\left(n+\tau_{2}\right) x^{\Delta}\left(n+\tau_{2}\right)\right)^{\Delta}}{x(\delta(n))}+\frac{\eta^{\Delta}(n)}{\eta(\sigma(n))} v(\sigma(n))-\frac{\eta(n)}{r(\delta(n)) \eta^{2}(\sigma(n))} v^{2}(\sigma(n))$.

Combining (25), (27) and (29), and using (4), we obtain

$$
\begin{gathered}
\omega^{\Delta}(n)+a_{0} u^{\Delta}(n)+b_{0} v^{\Delta}(n) \\
\leq-\eta(n) Q(n)+\frac{\eta^{\Delta}(n)}{\eta(\sigma(n))} \omega(\sigma(n))-\frac{\eta(n)}{r(\delta(n)) \eta^{2}(\sigma(n))} \omega^{2}(\sigma(n)) \\
+a_{0} \frac{\eta^{\Delta}(n)}{\eta(\sigma(n))} u(\sigma(n))-a_{0} \frac{\eta(n)}{r(\delta(n)) \eta^{2}(\sigma(n))} u^{2}(\sigma(n)) \\
+b_{0} \frac{\eta^{\Delta}(n)}{\eta(\sigma(n))} v(\sigma(n))-b_{0} \frac{\eta(n)}{r(\delta(n)) \eta^{2}(\sigma(n))} v^{2}(\sigma(n)) .
\end{gathered}
$$

Applying operator $T$ to (30), and from (21), we have

$$
\begin{gathered}
T[\eta(s) Q(s) ; l, n] \\
\leq T\left[\left(\psi(n, s, l)+\frac{\eta^{\Delta}(s)}{\eta(\sigma(s))}\right) \omega(\sigma(s))-\frac{\eta(s)}{r(\delta(s)) \eta^{2}(\sigma(s))} \omega^{2}(\sigma(s))\right. \\
+a_{0}\left(\psi(n, s, l)+\frac{\eta^{\Delta}(s)}{\eta(\sigma(s))}\right) u(\sigma(s))-a_{0} \frac{\eta(s)}{r(\delta(s)) \eta^{2}(\sigma(s))} u^{2}(\sigma(s)) \\
\left.+b_{0}\left(\psi(n, s, l)+\frac{\eta^{\Delta}(s)}{\eta(\sigma(s))}\right) v(\sigma(s))-b_{0} \frac{\eta(s)}{r(\delta(s)) \eta^{2}(\sigma(s))} v^{2}(\sigma(s)) ; l, n\right]
\end{gathered}
$$

By the averaging technique, from (31), we obtain

$$
\begin{gathered}
T[\eta(s) Q(s) ; l, n] \leq T\left[\frac{\left(1+a_{0}+b_{0}\right)}{4 \eta(s)}(\psi(n, s, l)\right. \\
\left.\left.+\frac{\eta^{\Delta}(s)}{\eta(\sigma(s))}\right)^{2} r(\delta(s)) \eta^{2}(\sigma(s)) ; l, n\right],
\end{gathered}
$$

that is

$$
\begin{gathered}
T[\eta(s) Q(s) \\
\left.-\frac{\left(1+a_{0}+b_{0}\right)}{4 \eta(s)}\left(\psi(n, s, l)+\frac{\eta^{\Delta}(s)}{\eta(\sigma(s))}\right)^{2} r(\delta(s)) \eta^{2}(\sigma(s)) ; l, n\right] \leq 0,
\end{gathered}
$$

which a contradiction to (22).

Next, assume case (ii) hold. Define $\omega, u$ and $v$ as (5), (7) and (9), proofing as in Theorem 1, we obtain inequality (15). Applying operator $T$ to (15), and from (21), we have

\section{$T[Q(s) ; l, t] \leq$}

$T\left[\psi(n, s, l) \omega(\sigma(s))+a_{0} \psi(n, s, l) u(\sigma(s))+b_{0} \psi(n, s, l) v(\sigma(s)) ; l, n\right]$

$$
-T\left[\frac{\omega^{2}(s)}{r(s)}+a_{0} \frac{u^{2}(s)}{r(s)}+b_{0} \frac{v^{2}(s)}{r(s)} ; l, n\right] .
$$

Since the decreasing property of $\omega(n), u(n)$ and $v(n)$, from (32) we have

$$
\begin{aligned}
& T[Q(s) ; l, n] \leq T\left[\psi(n, s, l) \omega(s)+a_{0} \psi(n, s, l) u(s)\right. \\
& \left.+b_{0} \psi(n, s, l) v(s)-\frac{\omega^{2}(s)}{r(s)}-a_{0} \frac{u^{2}(s)}{r(s)}-b_{0} \frac{v^{2}(s)}{r(s)} ; l, n\right] .
\end{aligned}
$$

By the averaging technique, we obtain

$$
T\left[Q(s)-\frac{\left(1+a_{0}+b_{0}\right)}{4} \psi^{2}(n, s, l) r(s) ; l, n\right] \leq 0,
$$

which a contradiction to (23). So the theorem is established.

Remark 2.1. We can obtain kinds of theorems of equation (1) by from Th 2 different choices of $\eta$ and $\varphi$.

Theorem 3. Assume that

$B(\delta(n))=\left(1-a_{0}-b_{0} \frac{R\left(\delta(n)+\tau_{2}\right)}{R(\delta(n))}\right)>0$, and positive function $\xi \in C_{r d}^{1}\left(\left[n_{0}, \infty\right)_{T}, R\right)$ exist to make $\frac{\xi(n)}{\phi(n) r(n)}+\xi^{\Delta}(n) \leq 0$ and $C(\delta(n))=\left(1-a_{0} \frac{\xi\left(\delta(n)-\tau_{1}\right)}{\xi(\delta(n))}-b_{0}\right)>0$, where $R(n)=\int_{n_{0}}^{n} \frac{1}{r(s)} \Delta s$ and $\phi(n)$ is defined as in Theorem 1. If there exist positive function $\theta \in C_{r d}^{1}\left(\left[n_{0}, \infty\right)_{T}, R\right)$ then

$$
\limsup _{n \rightarrow \infty} \int_{n_{2}}^{n}\left[\theta(s) q(s) B(\delta(s))-\frac{\left(\theta^{\Delta}(s)\right)^{2} r(\delta(s))}{4 \theta(s)}\right] \Delta s=\infty .
$$

And

$$
\limsup \int_{n \rightarrow \infty}^{n}\left[\phi(s) q(s) C(\delta(s))-\frac{1}{4 \phi(s) r(s)}\right] \Delta s=\infty .
$$

Then equation (1) oscillation.

Proof. Suppose that $y$ is a eventually positive solution of (1). Yet the general, we may assume that $n_{1} \geq n_{0}$ exists to make $y(n)$, $y\left(n-\tau_{1}\right), y\left(n+\tau_{2}\right)$ and $y(\delta(n))$ are positive for $n \in\left[n_{1}, \infty\right)_{T}$.

Proof steps similar to Theorem 1, for $n \in\left[n_{2}, \infty\right)_{T}$ 
we have case (i) and case (ii).

Firstly, assume that case(i) hold. Since $r(n) x^{\Delta}(n)<0$, then

$$
r(s) x^{\Delta}(s) \geq r(n) x^{\Delta}(n), n \geq s \geq n_{1} \text {. }
$$

Through calculation, we have

$$
x(n) \geq R(n) r(n) x^{\Delta}(n) .
$$

Then, we obtain

$$
\begin{aligned}
& \left(\frac{x(n)}{R(n)}\right)^{\Delta}=\frac{x^{\Delta}(n) R(n)-x(n) R^{\Delta}(n)}{R(n) R(\sigma(n))} \\
& =-\frac{1}{r(n)}\left[\frac{x(n)-r(n) x^{\Delta}(n) R(n)}{R(n) R(\sigma(n))}\right] \leq 0 .
\end{aligned}
$$

Since $\frac{x(n)}{R(n)}$ is nonincreasing, then

$$
x\left(n+\tau_{2}\right) \leq \frac{R\left(n+\tau_{2}\right)}{R(n)} x(n) .
$$

From $x(n)$ and (35), we have

$$
\begin{array}{r}
y(n) \geq x(n)-a_{0} x\left(n-\tau_{1}\right)-b_{0} x\left(n+\tau_{2}\right) \\
\geq\left(1-a_{0}-b_{0} \frac{R\left(n+\tau_{2}\right)}{R(n)}\right) x(n) .
\end{array}
$$

Combining (1) with (35), then

$$
\left(r(n) x^{\Delta}(n)\right)^{\Delta}+q(n) B(\delta(n)) x(\delta(n)) \leq 0 .
$$

Define function

$$
\omega(n)=\theta(n) \frac{r(n) x^{\Delta}(n)}{x(\delta(n))} n \in\left[n_{2}, \infty\right)_{T} .
$$

Obviously, $\omega(n)>0$. Differentiating $\omega(n)$, and from (37), (38), we obtain

$$
\omega^{\Delta}(n)
$$$$
=\frac{\theta(n)}{x(\delta(n))}\left(r(n) x^{\Delta}(n)\right)^{\Delta}+\frac{\theta^{\Delta}(n)}{\theta(\sigma(n))} \omega(\sigma(n))-\frac{\theta(n) x^{\Delta}(\delta(n))}{\theta(\sigma(n)) x(\delta(n))} \omega(\sigma(n))
$$$$
\leq-\theta(n) q(n) B(\delta(n))+\frac{\theta^{\lrcorner}(n)}{\theta(\sigma(n))} \omega(\sigma(n))-\frac{\theta(n)}{r(\delta(n)) \theta^{2}(\sigma(n))} \omega^{2}(\sigma(n)) .
$$

By the averaging technique, from (39), so

$$
\omega^{\Delta}(n) \leq-\theta(n) q(n) B(\delta(n))+\frac{\left(\theta^{\Delta}(n)\right)^{2} r(\delta(n))}{4 \theta(n)} .
$$

Integrating both sides of above form from $n_{2}$ to $n$, we obtain

$$
\int_{n_{2}}^{n}\left[\theta(s) q(s) B(\delta(s))-\frac{\left(\theta^{\Delta}(s)\right)^{2} r(\delta(s))}{4 \theta(s)}\right] \Delta s \leq \omega\left(n_{2}\right),
$$

which a contradiction to (33).
Next, assume case (ii) holds. Since $r(n) x^{\Delta}(n)$ is decreasing, we have

$$
r(s) x^{\Delta}(s) \leq r(n) x^{\Delta}(n), s \geq n \geq n_{2} .
$$

Through calculation, we have

$$
x(l) \leq x(n)+r(n) x^{\Delta}(n) \int_{n}^{l} \frac{1}{r(s)} \Delta s .
$$

When $l \rightarrow \infty$, we obtain

$$
0 \leq x(n)+\phi(n) r(n) x^{\Delta}(n) .
$$

Then, for $\xi(n)$, we have

$$
\begin{gathered}
\left(\frac{x(n)}{\xi(n)}\right)^{\Delta}=\frac{x^{\Delta}(n) \xi(n)-x(n) \xi^{\Delta}(n)}{\xi(n) \xi(\sigma(n))} \\
\geq-\frac{x(n)}{\xi(n) \xi(\sigma(n))}\left(\frac{\xi(n)}{\phi(n) r(n)}+\xi^{\Delta}(n)\right) \geq 0
\end{gathered}
$$

Hence, we obtain

$$
y(n) \geq\left(1-a_{0} \frac{\xi\left(n-\tau_{1}\right)}{\xi(n)}-b_{0}\right) x(n) .
$$

Combining (1) with (41), we conclude

$\left(r(n) x^{\Delta}(n)\right)^{\Delta}+q(n) C(\delta(n)) x(\delta(n)) \leq 0$.

Define function

$$
v(n)=\frac{r(n) x^{\Delta}(n)}{x(n)} n \in\left[n_{2}, \infty\right)_{T} .
$$

Obviously, $v(n)<0$. From (40) and (43), we can obtain

$$
-1 \leq \phi(n) v(n) \leq 0
$$

Differentiating (43), and from (42), we conclude

$$
v^{\Delta}(n) \leq-q(n) C(\delta(n))-\frac{v^{2}(n)}{r(n)} .
$$

Then $v^{\Delta}(n)<0$. Multiplying both side of above inequality by $\phi(n)$ and integrating from $n_{2}$ to $n$, using the integration by parts on time scales, we have

$$
\begin{gathered}
\phi(n) v(n)-\phi\left(n_{2}\right) v\left(n_{2}\right)-\int_{n_{2}}^{n} \frac{1}{r(s)} v(s) \Delta s \\
+\int_{n_{2}}^{n} \phi(s) q(s) C(\delta(s)) \Delta s+\int_{n_{2}}^{n} \frac{\phi(s)}{r(s)} v^{2}(s) \Delta s \leq 0 .
\end{gathered}
$$

By the averaging technique, and from (44), we obtain

$$
\int_{n_{2}}^{n}\left[\phi(s) q(s) C(\delta(s))-\frac{1}{4 \phi(s) r(s)}\right] \Delta s \leq 1+\phi\left(n_{2}\right) v\left(n_{2}\right),
$$


which a contradiction to (34). The proof is now completed.

\section{Examples and summary}

In this section, some examples are presented to explain the significance of our harvest.

Example 3.1. Consider

$$
\left(t^{2} x^{\Delta}(n)\right)^{\Delta}+3 n y(n-c)=0, n \in[1, \infty)_{T},
$$

where $x(n)=y(n)+y(n-c)+2 y(n+c), c>0$ is constant.

Here $r(n)=n^{2}, a(n)=1, b(n)=2, q(n)=3 n$, $\tau_{1}=\tau_{2}=c, \delta(n)=n-c$.

Then $\quad \delta(n \pm \alpha)=\delta(n) \pm \alpha(\alpha>0) \quad$ holds, $Q(n)=3(n-c), \phi(n)=\int_{n}^{\infty} \frac{1}{s^{2}} \Delta s$, and it is not hard to verify that (2), (3) are satisfied. Therefore, equation (45) oscillation because of Theorem 1 .

Example 3.2. Consider the following dynamic equation

$$
\left(t^{2} x^{\Delta}(n)\right)^{\Delta}+\frac{1}{(n-c)^{2}} y(n-c)=0, n \in[1, \infty)_{T},
$$

where $x(n)=y(n)+y(n-c)+2 y(n+c), c>0$ is constant. Here $r(n)=n^{2}, a(n)=1, b(n)=2, q(n)=3 n, \tau_{1}=\tau_{1}=c, \delta(n)=n-c$. Then $\delta(n \pm \alpha)=\delta(n) \pm \alpha(\alpha>0) \quad$ and $\quad \delta(n) \leq n-\tau_{1} \quad$ hold, $\quad Q(n)=\frac{1}{n^{2}}$ Choosing $\varphi(n, s, l)=(n-s)(s-l)(n \geq s \geq l \geq 1)$, then $\varphi(n, s, l)$ belongs to function class $Y$, and $\varphi^{\Delta_{s}}(n, s, l)=n-2 s+l$, and from (20) we have $\psi(n, s, l)=\frac{n-2 s+l}{(n-s)(s-l)}$. It is apt to educe that (22) and (23) established. Therefore, (46) is oscillatory due to Theorem 2.

\section{Acknowledgements}

This artical is sustained by the Natural Science Foundation of China (617043180), and sustained by Shandong Provincial Natural Science Foundation (ZR2016AM17, ZR2017MA043).

\section{References}

1. R. P. Agarwal, D. O'Regan, S H Saker, Oscillation criteria for second-order nonlinear neutral delay dynamic equations, J. Math Anal. and Appl., doi:10.1016/j.jmaa.2004.06.041.

2. S. Hilger, Analysis on measure chains - a unified approach to continuous and discrete calculus, Results Math., 1990, 18 (1-2): 18-56.

3. S. H. Saker, D. O'Regan, New oscillation criteria for second-order neutral dynamic equations on time scales via Riccati substitution. Hiroshima Mathematical Journal, 2012, 42(2012):77-98.

4. R. Wang, Q. Li, Oscillation and asymptotic properties of a class of second-order Emden-Fowler neutral differential equations, Springerplus, 2016, 5(1): 1956 .

5. B. Karpuz, Sufficient Conditions for the Oscillation of Odd-Order Neutral Dynamic Equations, International Journal of Analysis and Applications, 2017, 14 (1): 69-76.

6. T. Li, Z. Han, S. Sun, D. Yang, Existence of nonoscillatory solutions to second-order neutral delay dynamic equations on time scales, Adv. Differ. Equ. 2009 (2009), Article ID 562329, 1-10.

7. Gaihua Gui Z X. Oscillation criteria for second-order neutral differential equations with distributed deviating arguments. Electronic Journal of Differential Equations, 2007, 2007(3):249-266.

8. Y. Bai, L. Liu. New Oscillation Criteria for SecondOrder Neutral Delay Differential Equations with Positive and Negative Coefficients, Abstract \& Applied Analysis, 2014, 2010(2-4):331-336.

9. Q. Hao, F. Lu, Oscillation theorem for superlinear second order damped differential equations, Appl. Math. Comput, 217 (2011) 7126-7131.

10. Y. Bolat, Oscillation criteria for nonlinear secondorder difference equations with a nonlinear damped term, Appl. math. Lett. 18 (2005) 329-338.

11. J. Jiang, X. Li, Oscillation of second order nonlinear neutral differential equations, Appl. Math. Comput, 135 (2003) 531-540.

12. Y. Sui, and S. Sun, Oscillation of third order nonlinear damped dynamic equation with mixed arguments on time scales, Adv. Diff. Equ., (2018) 2018:233 DOI.org/10.1186/s13662-018-1654-3.

13. Y. Sui, Z. Han, Oscillation of third-order nonlinear delay dynamic equation with damping term on time scales, J. Appl. Math. Comput. (2018) 58, DOI.org/10.1007/s12190-017-1158-4. 